Thorax (1961), 16, 192.

\title{
HETEROTOPIC MEDIASTINAL THYROID
}

\author{
BY \\ B. T. LE ROUX \\ From the Departments of Surgery, University of Edinburgh
}

(RECEIVED FOR PUBLICATION MAY 30, 1960)

Developmental aberrations of the thyroid gland are common. It develops from pharyngeal endoderm, appearing in the fourth embryonic week as a hollow median outgrowth from the floor of the primitive pharynx at the site of the future foramen caecum of the tongue. The thyroid rudiment lies in intimate relationship with the aortic sac, being in direct contact with its endothelium. The rudiment grows downwards on a hollow stalk, the thyroglossal duct or canal of His, in close relationship with tissues which will later form the body of the hyoid bone. The lingual end of the thyroglossal duct persists as the foramen caecum. Functionally active aberrant thyroid tissue may be found at the base of the tongue, along the normal course of the thyroglossal duct, in the anterior mediastinum, in the pericardium, or in the heart (Willis, 1958). Heterotopic thyroid in the mediastinum, pericardium, and heart has probably grown from rudiments of developing thyroid dragged into the chest during descent of the heart and great vessels with the development of the embryonic neck and the unfolding of the embryo.

Heterotopic thyroid tissue in the anterior mediastinum usually lies in the vicinity of the thymus, and may or may not have an obvious connexion with the normally situated cervical thyroid. Mediastinal heterotopic goitre is an entity distinct from retrosternal extension of one or other of the inferior poles of a cervical goitre. Heterotopic thyroid in the mediastinum may derive its blood supply locally or from vessels originating in the neck. It may on rare occasions be the only functioning thyroid tissue.

The patient harbouring heterotopic thyroid tissue in the mediastinum is usually symptomless, and presents because of the finding of an anterior mediastinal opacity on a chest radiograph made for unrelated reasons. The diagnosis of heterotopic thyroid is unlikely to be considered. The common lesions in the anterior mediastinum are teratomata and thymic tumours, and it is with either of these diagnoses that thoracotomy is likely to be undertaken. Preliminary investigations are unlikely to have been of value unless they included the exhibition of radioactive iodine and scanning of the mediastinum. The value of this manœuvre has been demonstrated by Swedenburg, Tuttle, and Corrigan (1955), who identified 10 anterior mediastinal lesions as thyroid in this way.

Heterotopic mediastinal thyroid is not rare, relative to other anterior mediastinal tumours. Three examples have been found over a decade in the Regional Thoracic Unit in Edinburgh, which subserves a population of about $1 \frac{1}{2}$ million. During the same period only 21 anterior mediastinal teratomata were found, and slightly fewer tumours of thymic origin, most of which were benign. Details from the three patients found to have heterotopic mediastinal thyroids are presented. In none was the diagnosis suspected before thoracotomy. All are well following resection. Two have been shown to have normal thyroid tissue in the neck, and the third is clinically euthyroid 10 years after resection of the mediastinal tumour.

\section{CASE REPORTS}

CASE 1.-As a preliminary to admission to a foreign country a 62-year-old woman was required to produce a chest radiograph. On this an opacity was seen in the anterior mediastinum; most of the shadow projected to the right (Figs. 1 and 2). There were no clinical abnormalities; fluoroscopy, bronchoscopy, and examination of peripheral blood were uninformative. With the provisional diagnosis of thymic tumour or teratoma right thoracotomy was undertaken in April, 1959.

A tumour, roughly spherical and about $6 \mathrm{~cm}$. in diameter, lay in the mediastinum between the trachea and the superior vena cava. In close proximity to the tumour were two $1.5 \mathrm{~cm}$. glands. Both the tumour and the glands were shelled cleanly out of the mediastinum after the incision of the overlying pleura. 


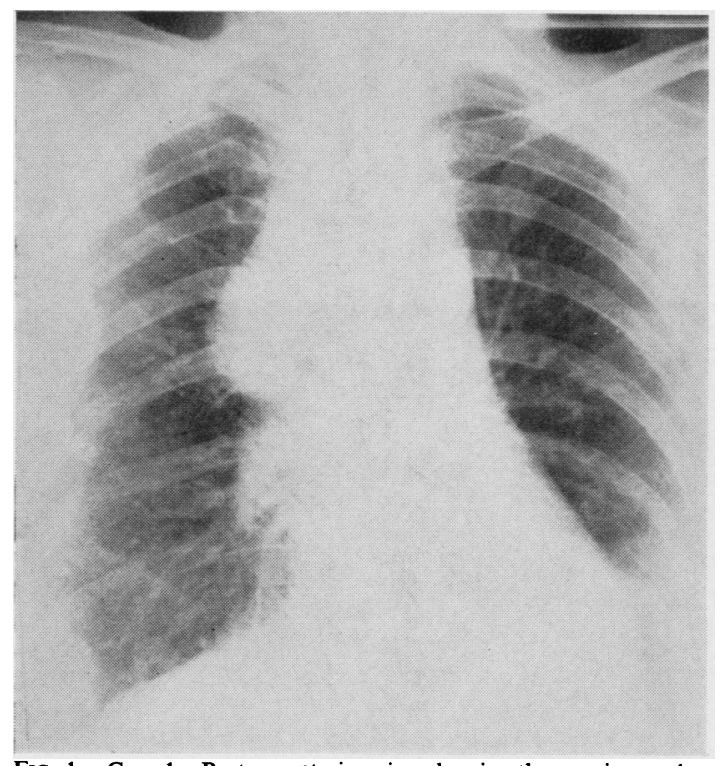

Fig. 1.-Case 1: Postero-anterior view showing the opacity on the right of the mediastinum.

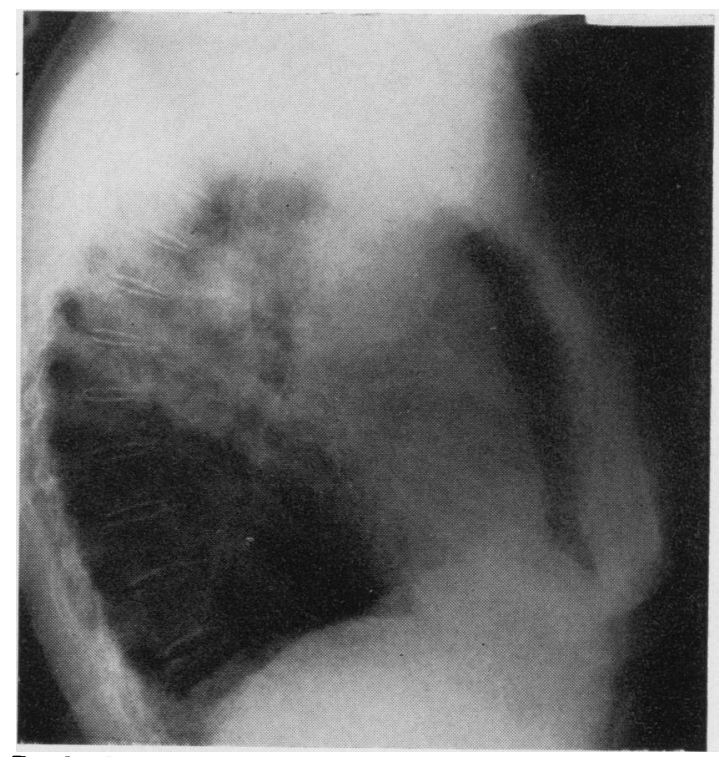

FIG. 2.-In the lateral view the opacity lies in front of and above the pulmonary hilum.

Fig. 3-Vacuolated macrophages and cholesterol crystals have accumulated in a focus of cystic degeneration. Haematoxylin and eosin, $\times 70$.

Fig. 4.-Case 2: Postero-anterior view showing the opacity obscuring the left pulmon ary hilum, but also projecting a little to the right of the mediastinum.

Fig. 5.-In the lateral view the opacity lies in the anterior mediastinum and rather lacks definition.

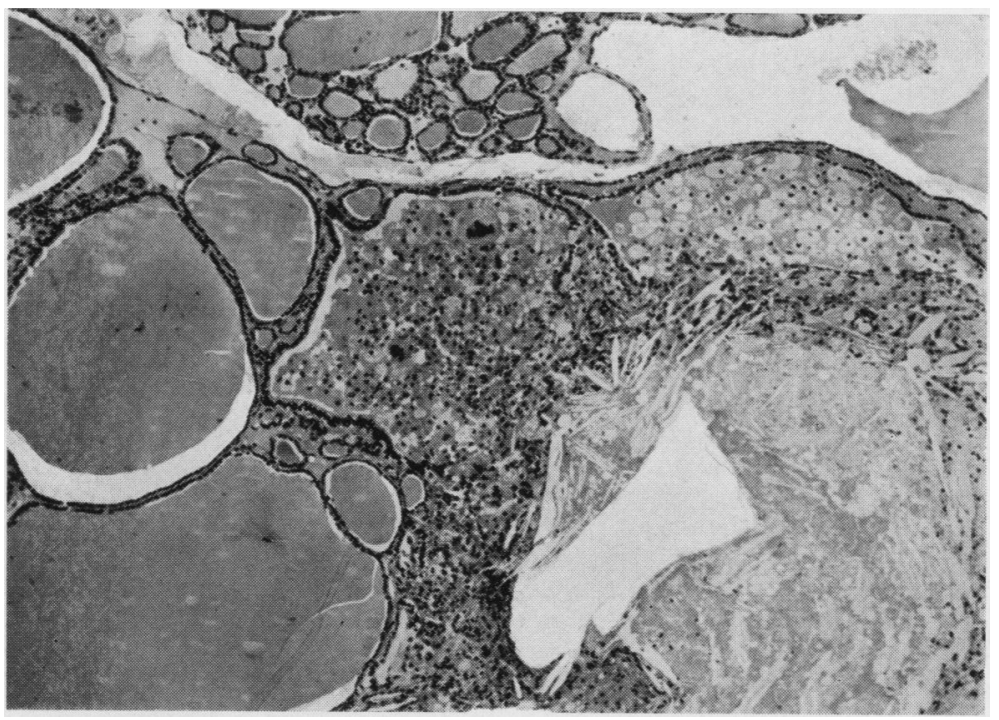

Fig. 3

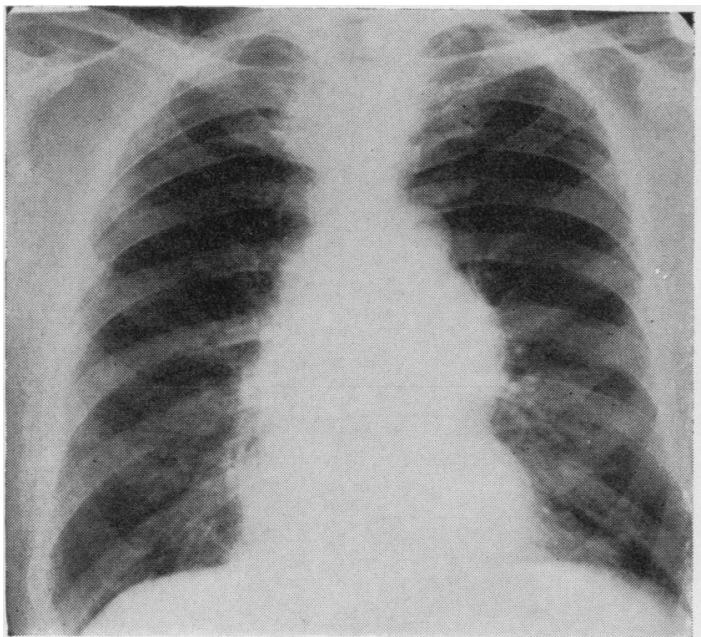

Fig. 4

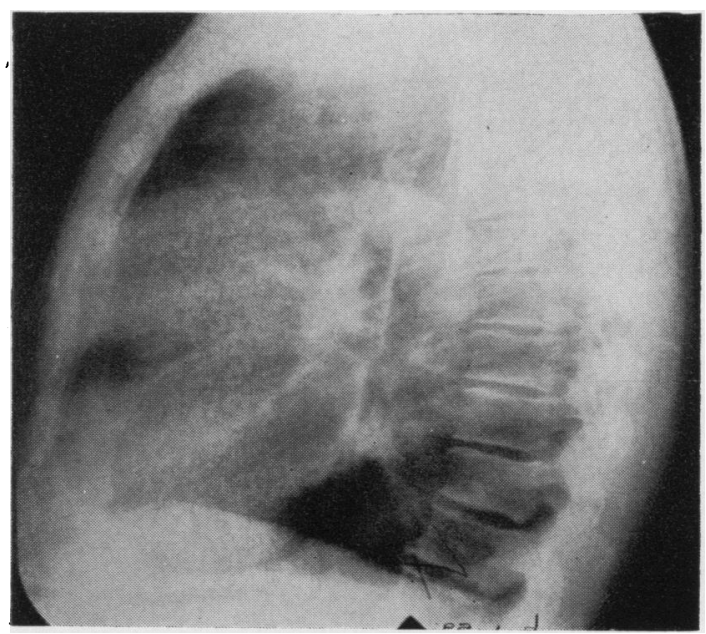

Fig. 5 
Only one small vessel coming from the vicinity of the thymus required ligation. Convalescence was uneventful. The tumour showed the histological features of colloid goitre with areas of extensive cystic degeneration (Fig. 3). The glands were found to show the features of sarcoidosis. The patient remains well, and a year after resection of the mediastinal mass shows none of the stigmata of sarcoidosis. The sarcoid reaction in the glands has therefore been attributed to the degenerative changes in the tumour. Post-operative radioactive iodine excretion tests and direct scanning of the neck have shown the patient to have a normal complement of thyroid tissue in the normal situation.

CASE 2.-A 67-year-old labourer was radiographed during the course of an acute respiratory illness. He was found to have a left basal opacity which cleared rapidly on subsequent films. In addition, a mass was seen in the anterior mediastinum, projecting mainly to the left and a little to the right of the mediastinum (Figs. 4 and 5). There were no related clinical abnormalities. The bronchoscopic appearances were normal ; fluoroscopy was uninformative ; the electrocardiogram was normal and no abnormality of peripheral blood was observed. An angiogram was made to exclude aneurysm of the aorta, and this showed a normal aortic outline. A provisional diagnosis of anterior mediastinal teratoma or thymic tumour was made ; in January, 1959, left thoracotomy was undertaken. The incision was extended medially to cross the sternum to facilitate access to a mass $10 \mathrm{~cm}$. in diameter overlying the ascending aorta. The mass was pulsatile and it was thought that it might yet be an aneurysm. The pericardium was opened to permit a decision on this point. The tumour was densely adherent to the aortic sheath, but from within the pericardium the sheath was easily stripped from the aorta, leaving the tumour attached only by a pedicle the cephalad extent of which was not accurately determined, and which may have reached as high as the neck. The pedicle was dissected and in it were found a little fat, some small veins, and one large artery with a lumen $3 \mathrm{~mm}$. in diameter. When this was ligated the mass ceased to pulsate. Convalescence from thoracotomy was uneventful.

The tumour showed the histological features of normal thyroid gland with one large colloid cyst. The patient remains well a year later. He is euthyroid, and neck scanning and radioactive iodine excretion tests show him to have a functioning thyroid gland in the normal site.

CASE 3.-A housewife of 49 presented with a story of intermittent cough for nine years, for seven of which she had been known to have an opacity in the anterior mediastinum. The opacity was increasing in size, and she was therefore advised to undergo thoracotomy. In its vertical extent the opacity measured $12 \mathrm{~cm}$.; it reached from the diaphragm to the ascending aorta, and projected to the left (Figs. 6 and 7). The only clinical abnormality was an area of dullness anteriorly, corresponding to the opacity.

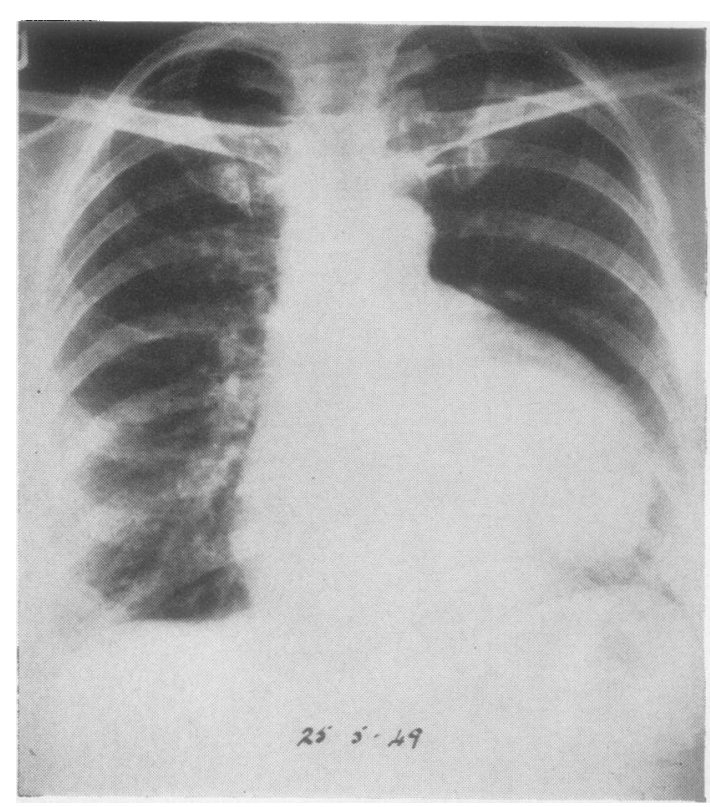

FIG. 6.-Case 3: Postero-anterior view showing the large opacity extending from the diaphragm to the aortic arch, and projecting to the left.

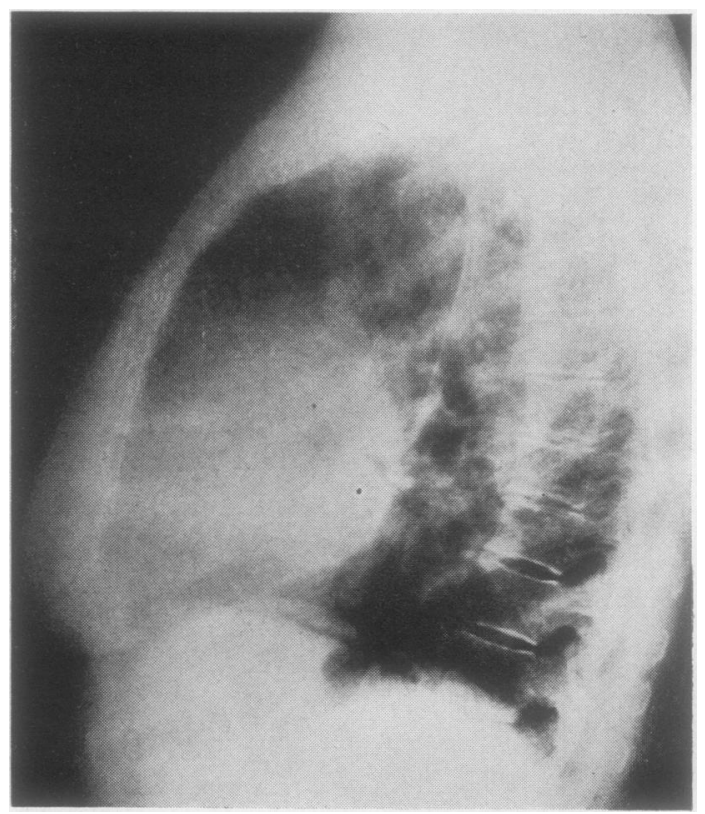

Fig. 7.-In the lateral view the opacity lies anteriorly. 


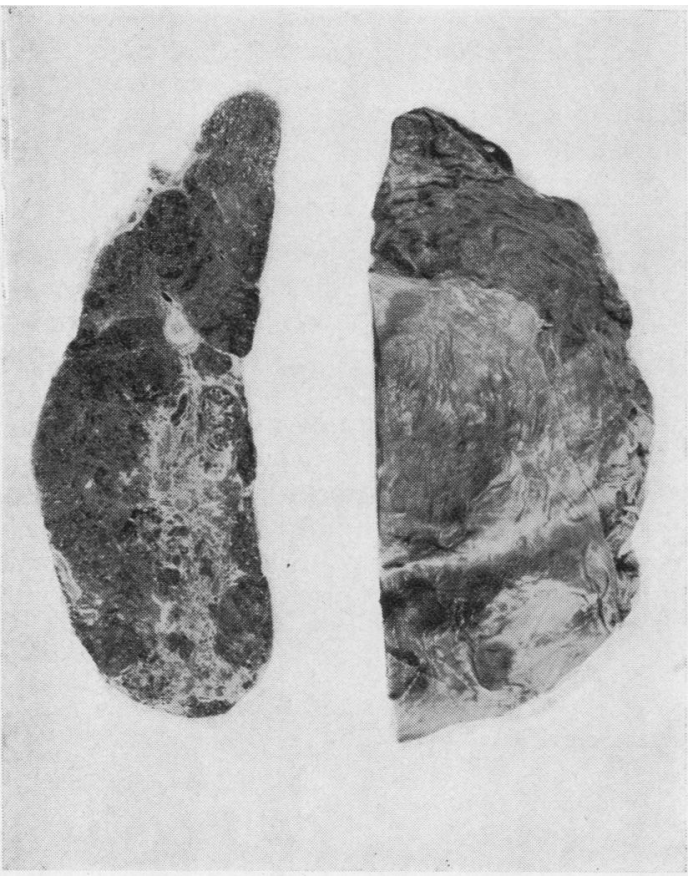

FIG. 8.-The specimen resected from Case 3.

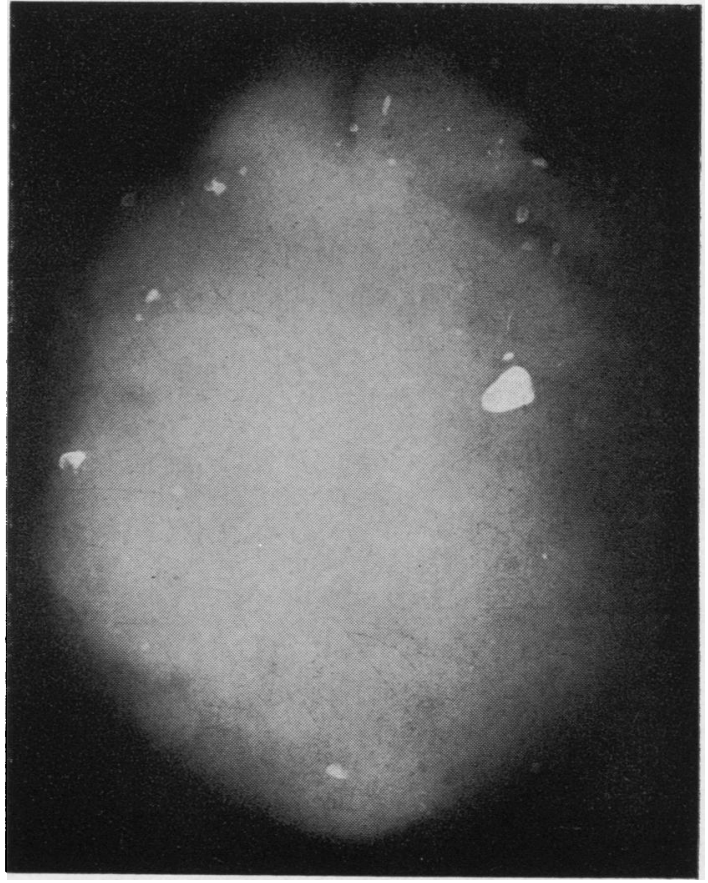

Fig. 9.-There is radiographic evidence of calcification within the resected specimen.

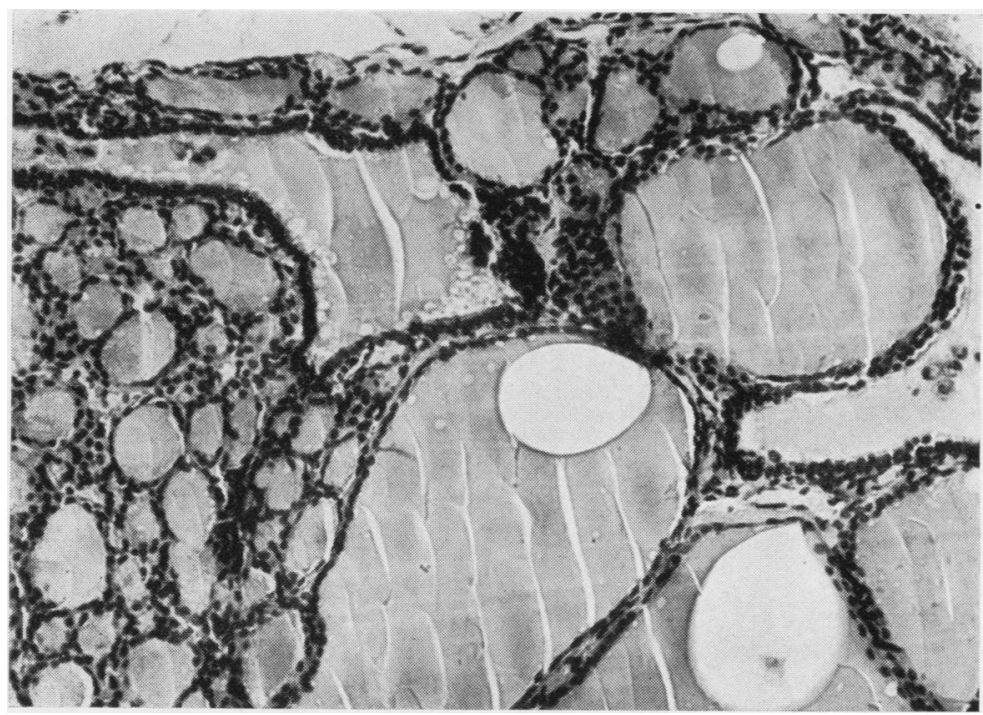

FIG. 10.-The acini vary in size and contain strongly eosinophilic colloid. Haematoxylin and cosin, $\times 175$. 
The bronchoscopic appearances were normal ; fluoroscopy was uninformative, and blood investigations were normal. The pre-operative diagnosis was anterior mediastinal teratoma ; the lesion was thought unlikely, from its size and relation to the diaphragm, to be a thymic tumour. Subsequent experience has shown these calculations to be erroneous.

In July, 1949, left thoracotomy was undertaken. A tumour 13 by 9 by $5 \mathrm{~cm}$. was enucleated from the anterior mediastinum after division of the pleural reflection (Fig. 8). The phrenic nerve was, however, adherent to the tumour, and, because there was uncertainty about the benignity of the lesion, the nerve was deliberately sacrificed and part of it was resected with the specimen. A small pedicle from the vicinity of the thymus was ligated. The tumour was soft and purple. Pre-operative radiographs suggested that there were within the tumour opacities of calcific density, and post-operative films of the tumour confirmed the presence of calcium within it (Fig. 9). Convalescence from thoracotomy was uneventful.

On cross-section the tumour had the typical appearances of a colloid goitre. There were numerous vesicles of varying sizes-some small, others 1-1.5 $\mathrm{cm}$. in diameter-and there were small areas of haemorrhage. Histologically the tumour was composed of thyroid tissue with acini varying in size from normal to large distended vesicles containing acidophil colloid and lined by cuboidal or flattened epithelium (Fig. 10), and was indistinguishable from a colloid goitre.

The patient is well 10 years after resection of the tumour. She is a little dyspnoeic, and this may be related to the height and paradoxical movement of the left dome of the diaphragm. Thyroid function has not been assessed; she is a vivacious Belgian woman with none of the features of myxoedema.

\section{SUMMARY}

Heterotopic thyroid must be included in the $\vec{\sigma}$ differential diagnosis of symptomless anterior iv mediastinal opacities; the commonest causes for such opacities are teratomata and thymic tumours. Functioning mediastinal thyroid tissue is sometimes recognizable pre-operatively by its absorption of radioactive iodine. This preliminary investigation may avoid the resection of such thyroid tissue should it prove to be the patient's only functioning thyroid.

\section{REFERENCES}

Swedenburg, R. D., Tuttle, W. M., and Corrigan, K. E. (1955). A.M.A. Arch. Surg., 71, 372.

Willis, R. A. (1958). The Borderland of Embryology and Pathology. Butterworths, London. 